\title{
Methodischer Ansatz zur Simulation wissenschaftlichen Schreibens im Fremdsprachenstudium: Vorgehensweise und Probleme bei Vermittlung und Erwerb wissenschaftlicher Textprozeduren
}

\author{
Simulating academic writing: \\ Didactic assumptions and problems in teaching \\ and following academic text procedures
}

\begin{abstract}
The preparation of a written diploma work is often a prerequisite for completion of one's academic studies. The requirements that it must comply with, require targeted and systematic instruction in developing academic writing skills. This article introduces the concept of academic writing and effects of developing it, based on the simulation of the editing processes of a scholarly text.

KEYWORDS: methodology of teaching writing, academic writing, a process-oriented approach, text procedures, title functions.
\end{abstract}

SCHLÜSSELWÖRTER: universitäres Schreiben, Schreibmethodik, Prozessorientierung, Textprozeduren, Titelfunktionen.

\section{HINFÜHRUNG ZUM THEMA UND ZIELSETZUNG}

Das Verfassen von Haus-, Seminar- oder Abschlussarbeiten stellt an vielen Universitäten weltweit einen obligatorischen Qualifikationsnachweis dar. Studierende müssen sich dabei an den Konventionen wissenschaftlicher Abhandlungen orientieren. In den zu produzierenden Texten haben Studierende unter Beweis zu stellen, dass sie sich mit einer Fragestellung reflektiert auseinandersetzen, ein bestimmtes Thema argumentativ entfalten und die formalen Standards wissenschaftlichen Arbeitens einhalten können (Steinhoff 2003: 39). Dazu gehören u.a. die Titelgebung, Textgliederung und deren Erläuterung, das Formulieren problemorientierter Leitfragen, ihre Einbettung in ein breiteres Forschungsgebiet, das Verfassen der Einleitung und des Schlussteils u.v.m. (Sandig 1997). 
Wegen des hohen kognitiven und sprachlichen Anspruchs gilt das wissenschaftliche Schreiben als die drittgrößte Schwierigkeit im Studium (Grieshammer 2011: 29). Daher ist es naheliegend, dass das wissenschaftliche Schreiben wegen dieser spezifischen Ansprüche einer gezielten und systematischen Unterstützung bedarf. Damit die Schreibkompetenz auf einem entsprechenden Niveau erworben werden kann, bietet man an den meisten Hochschulen Schreibkurse und entsprechende Lernmaterialien sowie Ratgeber mit praktischen Anleitungen an ${ }^{1}$. Deren Menge und Ausführlichkeit zeugt von der Relevanz und Komplexität des Problems. Es werden auch methodische Konzepte zu Entwicklung wissenschaftlichen Schreibens publiziert oder Aspekte fremdsprachlicher Textproduktion in der Wissenschaftssprache Deutsch eingehender diskutiert ${ }^{2}$.

Das Problem spitzt sich im Falle nichtmuttersprachlicher Studierender wegen ihrer nicht hinreichenden Sprach- und Schreibkompetenz sowie kultureller Unterschiede noch mehr $\mathrm{zu}^{3}$. Wie Analysen deutscher Lehrwerke für den Deutschunterricht als Fremdsprache von Thonhauser-Jursnick (2000: 197) und Fischer-Kania (2008: 492) zeigen, liegen die Ursachen für die defizitäre Schreibkompetenz u.a. darin, dass

- das kommunikativ-funktionale Schreiben im Fremdsprachenunterricht anhand einer stark beschränkten Anzahl von Textmodellen vermittelt,

- auf Vermittlung metakognitiver Schreibstrategien weitgehend verzichtet,

- und das textlinguistische Basiswissen zu Aspekten der grammatischen und semantischen Textkohärenz und Textsorten nicht gezielt aufgebaut wird (Iluk 2010: 24).

Folglich fehlt es den Studierenden an prozeduralem Wissen über die Gestaltung der einzelnen Textteile. Gleiches gilt für entsprechende Textroutinen. Zusammengestellte Listen mit Routineausdrücken, wie sie im Internet und auch Schreibratgebern zur Verfügung gestellt werden, erweisen sich als wenig hilfreich, da sie dekontextualisiert angeboten werden. Ihre produktive und angemessene Verwendung in zusammenhängenden Texten bedarf systematischer Übungen. Ohne diese bleiben wichtige Schreibstrategien und -fertigkeiten unterentwickelt (Iluk 2015: 102). Wenn dem so ist, so sollte es uns nicht verwundern, dass Schreiben zu den am wenigsten beliebten Lernhandlungen im Sprachstudium gezählt wird (Wysocka 1989: 106).

${ }^{1}$ Z.B. Pospiech (2004), Universität Essen; Rettig (2014), Campus Koblenz; Tipps zu Studientechniken und Lernmethoden (2011), Universität Bielefeld.

${ }^{2}$ Exemplarisch seien hier die Publikationen von G. Ruhmann (1997) und E. Venohr (2009, 2013) zu nennen.

${ }^{3}$ Aspekte eigenkulturellen Textsortenwissens und dessen interferierender Einfluss auf die Anfertigung schriftlicher Arbeiten in der Fremdsprache behandelt E. Venohr (2013) am Sprachenpaar Deutsch-Französisch. 
Im Folgenden soll ein methodischer Ansatz vorgestellt werden, mit dem man Studierende auf die Anfertigung ihrer Abschlussarbeit vorbreiten kann. Dem Ansatz liegt die Idee zugrunde, dass man das Verfassen einer wissenschaftlichen Arbeit zu Übungszwecken modellhaft und schrittweise nachbilden kann. Zunächst werden die Voraussetzungen erläutert, die eine solche Vorgehensweise begründen. Danach werden die Ergebnisse einer im Germanistikstudium der Schlesischen Universität in Katowice durchgeführten Schreibübung vorgestellt und eingehend diskutiert ${ }^{4}$.

\section{VORAUSSETZUNGEN FÜR EINE GELUNGENE SCHREIBSIMULATION}

In der Schreibsimulation geht es generell darum, einem vorgegebenen, kurzen Text die Struktur und die Merkmale einer wissenschaftlichen Arbeit zu geben $^{5}$. Bei dem hier konzipierten Schreibansatz ist Folgendes zu beachten:

- Im Sinne einer prozessorientierten Schreibdidaktik sollen schriftliche Aufgabenstellungen die Lerner weder kognitiv noch sprachlich überfordern. Ist das der Fall, so besteht die Gefahr, dass sich negative Emotionen und somit eine negative Einstellung zur Schreibaufgabe entwickeln. Diese binden bekanntlich kognitive Kapazitäten, so dass sie dann bei einer Aufgabenbearbeitung nicht mehr im ausreichenden Maß vorhanden sind. Sie wirken sich auch demotivierend auf die Bereitschaft zur aktiven Teilnahme an einer Aufgabe aus und folglich schmälern sie die zu erbringende Leistung.

- Schreibhandlungen in der Fremdsprache stellen besonders hohe Anforderungen, deshalb ist es sinnvoller, sie schrittweise vollziehen zu lassen. Die Kapazität des Arbeitsgedächtnisses setzt nämlich Grenzen, jenseits derer die kognitiven Prozesse bei der Textverarbeitung und -produktion sehr beeinträchtigt werden. Nach dem Prinzip des limitierten Aufmerksamkeitsquantums ist die optimale Aufmerksamkeitslenkung nur dann möglich, wenn sie auf nur einen Aktionsbereich fokussiert ist. Bei der Aufteilung der Aufmerksamkeit auf mehrere Aktionsebenen, z.B. Dekodierungspro-

${ }^{4}$ An dem Schreibseminar nahmen Studierende des Masterstudiums an der Germanistik mit dem Schwerpunkt Translatorik (Filologia germańska) teil, in dem sie auch ihre Abschlussarbeit zu verfassen hatten. Ihre Sprachkompetenz entsprach etwa dem Niveau B2 und darüber.

${ }^{5}$ Unter simulieren verstehen wir mit Duden: Vorgänge [...] modellhaft zu Übungs-, Erkenntniszwecken nachbilden, wirklichkeitsgetreu nachahmen. https:/ / www.duden.de/rechtschreibung/ simulieren [Zugriff am: 10.10.2020]. Demzufolge bedeutet Schreibsimulation eine Übungsform, in der die einzelnen Etappen der Anfertigung einer wissenschaftlichen Arbeit an einem vorgegebenen Text geübt werden, der keine Merkmale eines wissenschaftlichen Textes hat. 
zesse auf der Wortebene und Bildung von Makrooperationen (wie etwa bei der Umformung von Mikropropositionen in Makropropositionen) ist eine Qualitätsminderung der einzelnen Leistungen zu beobachten. Je mehr Gedächtniskapazitäten auf hierarchieniedrigeren Texterschließungsebenen in Anspruch genommen werden, umso weniger Aufmerksamkeit bleibt zur Ausführung von kognitiven Prozessen auf höheren Ebenen zur Verfügung (Karcher 1988: 215). Darüber hinaus verlaufen der lexikalische Zugriff wegen der intensiven Suchprozesse in der Fremdsprache sowie die Textproduktionsprozesse mühsamer und mit Verzögerung ${ }^{6}$. Die Folge ist, dass Studierende für kognitive Prozesse, wie etwa Analyse, Elaboration, Inferenzziehung, Generalisierung, Satzbildung, Evaluation in der Regel keine hinreichenden Ressourcen mehr übrig haben. Demzufolge schafft die Aufteilung einer Lernhandlung in einzelne Arbeitsschritte günstigere Voraussetzungen dafür, dass der kognitive Aufwand maximal auf die jeweils zu erbringende Teilleistung fokussiert werden kann. Auf diese Weise lassen sich das Risiko der Überforderung und die Überbelastung der kognitiven Ressourcen der Schreibenden minimieren.

- Wichtig für das Gelingen einer Schreibaufgabe ist deren kognitives und sprachliches Schwierigkeitsniveau. Wenn nötig, kann man dieses reduzieren, indem die zu erlernenden Teilfertigkeiten des wissenschaftlichen Schreibens an einem vorgegebenen fremdsprachlichen Ausgangstext erworben werden. Solch ein Text sollte inhaltlich und sprachlich relativ einfach und eher kurz sein, damit die Texterschließungsprozesse auf lexikalischer und syntaktischer Ebene die Aufmerksamkeit für die jeweiligen Formulierungshandlungen nicht beeinträchtigen. Die Arbeit an einem vorgegebenen Text hat also den Vorteil, dass sich Schreibende ausschließlich auf die zu vollziehenden, im folgenden Kapitel genannten Textprozeduren konzentrieren können.

\section{ZUM METHODISCHEN VORGEHEN}

Textprozeduren, wie Titelgebung, Textgliederung, Formulieren von Einleitung usw. lassen sich wirklichkeitstreu und modelhaft simulierend üben, wenn man, wie weiter unten gezeigt wird, kurze, ungegliederte, titellose,

${ }^{6}$ Die Ursachen für Leistungsgrenzen werden u.a. in den Merkmalen des kognitiven Systems und der ablaufenden Prozesse gesehen. Darauf haben direkten Einfluss die Menge an Einheiten, die ein individuelles System aufnehmen kann, ihre Haltedauer und Geschwindigkeit der Teilprozesse (Rummer, Mohr \& Zimmer 1988: 134). Die Auswirkungen der individuellen Leistungsbegrenzungen stehen auch im Zusammenhang mit dem Kompetenzniveau der jeweiligen Fremdsprache der Schreibenden einher. 
leicht verständliche Texte einsetzt. Dadurch entfällt die Belastung, einen Text zu einem vorgegebenen Thema selbst zu verfassen. Diese Bedingungen erfüllt der dem Lehrwerk Deutsch 2000 (Schäpers, Luscher \& Glück 1978: 63) entnommene Text:

Köln. Polizei rückte zum Schutz des Lufthansabüros an - und rückte wieder ab, als die Demonstrantinnen gar keinen Versuch machten, das Gebäude zu besetzen. Sie verteilten nur Flugblätter und diskutierten mit Passanten. Aufihren Spruchbändern stand "Männer fliegen - Frauen fliegen raus!" "Verfassungsfeinde in der Lufthansa!" und "Wir fordern Gleichberechtigung!" Die Frauenorganisationen waren auf die Straße gegangen, um gegen die Haltung der Deutschen Lufthansa zu protestieren, die es wieder einmal abgelehnt hatte, Frauen als Piloten einzuteilen. Konkreter Anlaß war die Absage an Rita Maiburg, die 40000 Mark in ihre Ausbildung als Pilotin gesteckt hatte und dann von der Lufthansa abgelehnt wurde. Nach Auffassung der Fluggesellschaft sind Frauen zu anfüllig, halten der Belastung nicht stand und sind im Hinblick auf die hohen Ausbildungskosten unrentabel, weil sie bald heiraten und dann ihren Beruf aufgeben.

Die dargestellte Geschichte ist nach dem Prinzip Folge-Ursache durchgehend entfaltet. Der rote Faden ist gut erkennbar, die logischen Beziehungen werden durch Konjunktionen ausgedrückt. Der Text besteht zum größten Teil aus dem Grundwortschatz. Allerdings gehören die Verben an- und abrücken zur Militärsprache und haben den Studierenden daher Verstehensprobleme bereitet ${ }^{7}$. Unbekannt waren auch die Ausdrücke anfällig und standhalten. Entscheidend für die Wahl des vorliegenden Textes war die Tatsache, dass man dessen thematische Entfaltung relativ leicht in eine chronologische Abfolge umwandeln kann. Durch diese Maßnahme begünstigt sollten die Schreibenden dafür sensibilisiert werden, dass man in einem Text Geschehnisse, Fakten, Handlungen nach einem bestimmten Prinzip ordnen und darstellen kann.

Die Studierenden konnten die jeweilige Teilaufgabe in Paaren realisieren. Die interaktive Arbeitsform ermöglichte es ihnen, sich frei auszutauschen und ihre Entscheidungen bzw. Formulierungsvorschläge miteinander zu diskutieren. Die Möglichkeit zur Interaktion zielt auf ein vertieftes Textverstehen und zugleich auf gegenseitige Animation zum Vollzug der jeweiligen Schreibhandlung sowie gemeinsame Evaluation. Die Übungsform hatte also einen stark prozessorientierten Ansatz. Die Erledigung der Schreibaufgaben in einzelnen, nacheinander zu vollziehenden Schritten bewahrte die Seminarteilnehmer vor kognitiver und sprachlicher Überforderung, die dann zustande kommt, wenn kognitive und sprachliche Entscheidungen simultan und ohne jegliche Hilfe zu treffen und zu vollziehen sind.

\footnotetext{
${ }^{7}$ Beide Verben begegnen uns auch häufig im Pressestil.
} 


\subsection{Arbeitsschritte}

Zum gezielten Übungsgegenstand wurden vier grundlegende Teilaufgaben gewählt:

a) chronologische Themenentfaltung,

b) Textgliederung,

c) Titelgebung,

d) Formulierung der Einleitung und des Schlussteils.

Nach der Erklärung der einzelnen Arbeitsschritte der Schreibaufgabe und deren Zielsetzungen im Schreibseminar begannen die Studierenden die jeweilige Teilaufgabe zu realisieren. Im Folgenden werden die einzelnen Schritte und ihre Zielsetzungen näher charakterisiert.

Schritt 1: Umwandlung des vorgegebenen Textes nach der chronologischen Abfolge der darin dargestellten Ereignisse und Fakten

Zunächst ging es darum, den Inhalt des Originaltextes nach seinem zeitlichen Verlauf umzustrukturieren. Mit der neuen Gliederung sollten die Ereignisse chronologisch dargestellt werden. Mit diesen Arbeitsschritt waren zwei Ziele verknüpft: Zum einen sollte den Studierenden klar werden, dass über ein und denselben Sachverhalt unterschiedlich berichtet werden kann. Die sich dabei ergebenden Differenzen in der Anordnung der Textinhalte gehen auf die angewandte Themenentfaltungsstrategie zurück. Damit die Studierenden bei der Textumwandlung nicht überfordert wurden, durften sie den Wortlaut des Ausgangstextes, soweit es möglich war, unverändert übernehmen. Zum anderen sollte den Seminarteilnehmern bewusst werden, dass bei der neuen Anordnung der Inhalte die gewählte Beschreibungsperspektive, die Zielsetzung, die logischen Relationen und Kohärenzprinzipien stets zu beachten sind. Die Bewusstmachung dieser Probleme ist wichtig, da Studierende bei der Anfertigung ihrer Abschlussarbeiten Informationen aus unterschiedlichen Quellen beziehen, die sie dann an ihre Themenentfaltungsstrategie anpassen und in den Text integrieren müssen.

Schritt 2: Textgliederung

Im nächsten Schritt sollte der umgewandelte Text in Sinneinheiten eingeteilt werden. Wegen der Textkürze haben die ausgesonderten Textteile den Umfang eines knappen Absatzes, manchmal sogar eines einzigen Satzes. Man kann sie daher als Quasi-Kapitel betrachten, denn in dieser Übung ging es nicht 
um die Einhaltung der üblichen Proportionen einzelner Textteile, sondern um die Entwicklung einer entsprechenden Sensibilität für eine kohärente Textgliederung.

Schritt 3: Titelgebung und Formulierung von Überschriften

$\mathrm{Zu}$ jedem ausgesonderten Textteil war in dieser Arbeitsphase eine passende Überschrift sowie zum gesamten Text der Haupttitel zu formulieren. In diesem Arbeitsschritt sollten dieStudierenden ihre Titelgebungskompetenz zeigen, wie sie die Gliederungsfunktion der Überschriften im Text nachvollziehen.

\section{Schritt 4: Verfassen von Einleitung und Schlussteil zum Text}

In der letzten Übungsphase waren die Einleitung und der Schlussteil zu dem so entstandenen Text zu formulieren. Zu diesem Zweck erhielten die Studierenden vom Seminarleiter entsprechende Information über deren Funktion und Struktur sowie entsprechende Routineformeln ${ }^{8}$. Damit der in Kapitel gegliederte Text die Regeln der Kohärenz nicht verletzt, mussten notwendige Ergänzungen hinzugefügt werden. In diesem Arbeitsschritt ging es darum, die Fähigkeit zu bestätigen, in einem Text das theoretische Wissen über die Funktionen einer Einleitung und des Schlussteils in die Praxis umzusetzen und die hierfür benötigten Routineformel produktiv zu gebrauchen'.

Aus der Beschreibung der Aufgabenstellung und der einzelnen Arbeitsschritte sollte für die Leser ersichtlich werden, dass es das generelle Lernziel war, an einem kurzen Text die Schreibetappen einer wesentlich längeren Arbeit, wie etwa einer Haus- oder Abschlussarbeit, und die dabei zu treffenden Entscheidungen aktiv und ohne größere Belastung nachzuvollziehen.

\section{EVALUATION DER ERGEBNISSE DER EINZELNEN ARBEITSSCHRITTE}

\subsection{Umwandlung der Themenentfaltungsstrategie}

Aufgrund der gewählten Themenentfaltungsstrategie der Originalfassung beginnt die Geschichte mit dem chronologisch letzten Ereignis, und zwar mit

\footnotetext{
${ }^{8}$ Diese Informationen wurden dem Leitfaden von Rettig (2004: 26-28) entnommen.

${ }^{9}$ Die Funktion und die Struktur der Einleitung und des Schlussteils in einem wissenschaftlichen Text wurden davor separat in einem anderen Seminar behandelt.
} 
dem Polizeieinsatz vor dem Lufthansa Büro in Köln, und endet mit der Begründung der Absage auf eine Bewerbung um eine Pilotenstelle durch die Fluggesellschaft.

Im umgewandelten Text waren dieselben Ereignisse in ihre chronologische Abfolge zu bringen. Zu diesem Zweck mussten die Studierenden die Episode identifizieren, mit der die erzählte Geschichte begann. Es erwies sich, dass das keine einfache Aufgabe war. Nach der Meinung fast aller Seminarteilnehmer war die Absage auf Bewerbung von R. Maiburg das primäre Ereignis. Dabei haben sie übersehen, dass die Protagonistin davor den Wunsch hatte, Pilotin zu werden, und deswegen eine entsprechende Ausbildung abschloss, für die sie eine hohe Gebühr bezahlte. Erst dann bewarb sie sich um eine Pilotenstelle bei der Lufthansa. Die Studierenden hatten also Probleme, das ursprüngliche Ereignis korrekt zu identifizieren. Daher wurde diese Aufgabe nicht ganz zufriedenstellend gelöst. Diese Arbeitsphase zeigte, dass die korrekte Themenentfaltung eine echte kognitive Herausforderung darstellt.

\subsection{Einteilung des Textes in Sinneinheiten}

Die Struktur wissenschaftlicher Arbeiten ist stark konventionalisiert. Ein wichtiges Element der Textkonventionen ist die Untergliederung in Kapitel und Unterkapitel, die durch Überschriften voneinander getrennt werden. Der Sinn der Textgliederung besteht darin, dass ein Thema in geordnete Einzelteile zerlegt wird, um so deren Sinnabschnitte zu verdeutlichen. Sie bilden die Grundlage für den Aufbau der Textstruktur. Die ausgewiesenen Einzelteile, wie etwa Kapitel, Unterkapitel, aber auch Absätze, müssen eine logische Einheit bilden und das behandelte Thema repräsentieren (Püschel 1997: 197). Deshalb ist die Textgliederung kein mechanischer, sondern ein analytischer und zugleich kreativer Prozess.

In dieser Schreibetappe bestand die Aufgabe darin, den umgewandelten Text in sachlogische Sinnabschnitte zu gliedern. Bei der Ausführung der Aufgabe wurden verschiedene Probleme erkennbar. Eine Ursache für die Schwierigkeit, den umgewandelten Text in selbständige Sinneinheiten zu gliedern, sah eine Seminarteilnehmerin in der Stringenz des Ausgangstextes.

Die Sätze sind u.a. wegen der Anwesenheit der Konjunktionen eng miteinander verbunden. Deswegen ist es schwierig, diesen Text in Abschnitte zu teilen. ${ }^{10}$

${ }^{10}$ Die Kommentare formulierte die Studentin spontan am Ende ihrer Schreibaufgabe. 
Es scheint also, dass in diesem Bereich ein latenter Übungsbedarf vorhanden ist. Die Seminarteilnehmerin erkannte aber gleichzeitig die Vorteile der vollzogenen Textgliederung für die Formulierung von Überschriften. Ihre Meinung begründete sie wie folgt:

Aber wenn man den Text schon eingeteilt hat, ist es einfach, die Überschriften für diese Abschnitte zu wählen, weil die Inhalte sehr konkret sind.

\subsection{Titelgebung}

Titel wissenschaftlicher Arbeiten sind keine bloßen Textbenennungen, sondern in der Regel eine Text- oder Textteilzusammenfassung. Sie sind das Ergebnis anspruchsvoller kognitiver und sprachlicher Verdichtungsprozesse (Gnutzmann 1988: 26). Die besondere Schwierigkeit bei deren Formulierung ergibt sich daraus, dass man mit relativ wenigen Wörtern und aufgrund struktureller Textraumbegrenzungen möglichst prägnant, viele Einzelheiten präzis mitteilen muss. Ähnliches gilt für die Formulierung von Einleitungen und Schlussfolgerungen. Die Funktionen der Haupttitel in wissenschaftlichen Arbeiten bestehen darin, dass sie

- als eine namensähnliche Kennzeichnung zu Identifikation und Wiedererkennung eines Textes fungieren,

- das Thema der Arbeit vorgeben,

- den Inhalt des Textes sachbezogen und prägnant wiedergeben oder auf einen besonderen Aspekt hindeuten,

- das Genre des Textes bezeichnen,

- das Leseinteresse wecken,

- als wichtige Lese- und Interpretationshilfe fungieren (Hellwig 1984: 6; Lüger 1987: 90-95; Gnutzmann 1988: 25; Dietz 1998: 618).

Die Zwischenüberschriften in einem Fließtext informieren dagegen über den nachfolgenden Textteil, verdeutlichen die Struktur und den thematischen Aufbau eines Textes. Haupttitel und vor allem Zwischen- und Untertitel erleichtern die Textverarbeitung, indem sie entsprechende Informationserwartungen evozieren und einen Zugriff zu bestimmten Inhalten ermöglichen (Iluk 2021: 9).

In der Fachliteratur wird zwischen thematischen und nicht-thematischen Titeln unterschieden. Die ersten repräsentieren Makropropositionen des Textes (Ballstaedt et al. 1981: 171). Nicht-thematische Überschriften in wissenschaftlichen Arbeiten beziehen sich auf die Superstruktur des Textes. Ihre Rolle besteht darin, dass sie den Zugang zu bestimmten Texteilen erleichtern. Beispiele für solche Titel sind: 
Einleitung, Zielsetzung, Fazit, Forschungsstand

Analysiert man die Titel in den umstrukturierten Texten, so fällt zunächst auf, dass die meisten die Form einer schwach attribuierten Nominalphrase aufweist, was dem Standard in wissenschaftlichen Arbeiten durchaus entspricht ${ }^{11}$. Gelegentlich begegnen uns auch satzförmige Titel. Dann haben sie die Form einer Frage, seltener eines Aussagesatzes.

Wie lief der Protest ab?

Wie haben die Frauen reagiert?

Gab es eine Konfliktlösung?

Die Frauen kämpfen für die Gleichberechtigung

Frauen protestieren

Nur wenige Texte wurden mit einem treffenden, vollthematischen Titel überschrieben.

Mangelnde Gleichberechtigung bei der Lufthansa als Grund für die feministische Demonstration

Frauendemonstration gegen Lufthansa in Köln

Formen des Kampfes um Gleichberechtigung von Frauen

In den meisten Texten wurden keine nichtthematischen Überschriften verwendet, obwohl sie eine Art Hinführung zum Thema, eine Information zum Textinhalt oder verbale Hinweise auf ein zu ziehendes Fazit enthalten. Daraus ist zu schließen, dass den Studierenden das Wissen über deren Funktion in einer wissenschaftlichen Arbeit fehlt oder beim Verfassen der Texte nicht darauf zugegriffen wurde. Wie zu erwarten war, zeigen die Haupttitel und Überschriften der einzelnen Textabschnitte typische Mängel, die generell darin bestehen, dass der Titelinhalt nicht mit dem Textinhalt übereinstimmt, d.h. der formulierte Titel ist thematisch umfangreicher als der Textinhalt, wie etwa:

Proteste wegen der Frauendiskriminierung bei Lufthansa

Das Problem des ungerechten Umgangs mit Frauen in der Deutschen Lufthansa

Demonstrationen in der Lufthansagesellschaft

Protest gegen die mangelnde Wertschätzung der Frauenrechte

oder Inhalte suggeriert, von denen im Ausgangstext nicht die Rede war, wie etwa

${ }^{11}$ Nach Ermittlungen von Kohrs (2010: 65) besteht fast die Hälfte deutscher eingliedriger Titel aus einer schwach attribuierten Nominalphrase. 
Misslungene Polizeiaktion in Köln

Staatsbehörden im Kampf gegen Sexismus

Formen des Kampfes um Gleichberechtigung von Frauen

Demonstrationsmethoden der Lufthansamitarbeiterinnen

Protestform der Pilotinnen

Können die Frauen wirklich über ihr Leben entscheiden?

Ein weiterer Mangel vieler Überschriften besteht darin, dass sie inhaltlich zu vage sind. Dieser Einwand gilt für Ein-Wort-Titel und semantisch elliptische Formulierungen.

Spruchbänder

Demonstration

Der Verlauf

Die Meinung der Lufthansa

Frauen protestieren

Zahlreiche Titel und Überschriften haben die Form einer Schlagzeile. Man kann hier den Einfluss der charakteristischen Titelgebung der Presse vermuten. An sich sind diese nicht falsch. Sie motivieren stärker zum Weiterlesen, aber sie informieren weniger über den eigentlichen Textinhalt. In dem Sinne sind sie keine Textparaphrasen, die in wissenschaftlichen Arbeiten zu erwarten sind.

Pilot - Traumberuf für alle?

Fliegen nicht für alle

Vergeudetes Geld für einen unerfüllbaren Traum

Die Geschichte von Rita Maiburg, die um Gleichberechtigung der Frauen und eigene Träume kämpfte

\subsection{Mängel in den Einleitungen}

Zunächst fällt auf, dass die Studierenden die Hinführung zum Thema mit einer Einleitung gleichsetzten, denn in diesem Textteil beschränkten sich die meisten auf die Andeutung des darzustellenden Themas.

Jeder weiß, dass Frauen früher um Gleichberechtigung kämpfen mussten, aber nicht alle kennen einzelne Geschichten von verschiedenen Revolutionärinnen. Eine von diesen und ihr Kampf wollte ich in dieser Arbeit vorstellen.

Die Folge ist, dass andere wesentliche Teile einer Einleitung unberücksichtigt geblieben sind. Es fehlen Angaben zu folgenden Aspekten: 
- Fragestellung und Zielsetzung der Arbeit,

- Abgrenzung / Eingrenzung des Themas,

- angewandte Methode / Vorgehensweise,

- Aufbau der Arbeit (Rettig 2004: 26).

Es ist naheliegend, dass bei einer derart konzipierten Schreibsimulation nicht alle Elemente einer wohlgeformten Einleitung vorkommen können. Dennoch wäre es zu erwarten gewesen, dass die genannten Bestandteile in der Einleitung in Ansätzen Erwähnung finden, wie etwa im folgenden Beleg.

Im Vordergrund der vorliegenden Arbeit steht das Problem des ungerechten Umgangs mit Frauen bei der Deutschen Lufthansa. Das Ziel der folgenden Arbeit ist es, die Ursachen und Folgen eines Konflikts darzulegen.

Es ist aber durchaus denkbar, dass zu Übungszwecken das Schreiben einer Einleitung mit allen wichtigen Elementen geübt werden kann.

\subsection{Typische Fehler}

Auf die sprachliche Korrektheit wird hier nicht eingegangen, da sie einem anderen Kompetenzbereich angehört und bei der Schreibübung nicht im Vordergrund stand. Außerdem könnte ein starker Fokus darauf die inhaltliche Formulierungskreativität wesentlich beeinträchtigen. Dennoch wollen wir auf zwei typische Fehler hinweisen, die regelmäßig in den von den Studierenden angefertigten Texten vorkommen. Die Titel und Überschriften stehen in einer sog. freistehenden Zeile. Daher darf hinter den Titel kein Punkt gesetzt werden (Grammis, \& 62) ${ }^{12}$. Diese Interpunktionsregel wird jedoch auch von den polnischen Studierenden häufig verletzt. Ein weiterer hochfrequenter Fehler ist der Artikelgebrauch. Haben die Titel und Überschriften eine nominale Form, so wird das einleitende Nomen in der Regel ohne Artikel gebraucht. In den umgewandelten Texten scheint diese Konvention (textueller Usus) unbekannt zu sein.

\subsection{Präsentation ausgewählter Texte}

Um sich ein vollständigeres Bild über die Ergebnisse der Schreibsimulation zu machen, werden im Folgenden zwei ausgewählte Texte präsentiert, die

${ }^{12}$ Siehe Grammis § 68. https://grammis.ids-mannheim.de/rechtschreibung/6200\#par68 [Zugriff am: 2.09.2020]. 


\begin{abstract}
u. E. die gesetzten und erreichten Lernziele gut illustrieren. Bei der Beurteilung dieser Schreibprodukte muss jedoch von den sprachlichen Fehlern abgesehen werden, denn - wie weiter oben bereits erwähnt wurde - stand die lexikalische und grammatische Korrektheit nicht im Vordergrund der Schreibsimulation.
\end{abstract}

\title{
Text 1
}

Das Problem des ungerechten Umgangs mit Frauen in der Deutschen Lufthansa

Im Vordergrund der vorliegenden Arbeit steht das Problem des ungerechten Umgangs mit Frauen in der Deutschen Lufthansa. Das Ziel der folgenden Arbeit ist es, die Ursachen und Folgen des Konflikts darzulegen.

\section{Ursprung des Konflikts}

Es soll im Folgenden veranschaulicht werden, woran die Ursache der Auseinandersetzung zwischen der Deutschen Lufthansa und der Pilotin Rita Maiburg liegt. Ein grundsätzliches Problem stellt die Arbeitsverweigerung der deutschen Fluglinien gegenüber Rita Maiburg. Unerlässlich in diesem Zusammenhang ist die Frage, ob eine Frau wegen ihres Geschlechts als Pilotin nicht eingestellt werden soll? Im Hinblick darauf muss allerdings hinzugefügt werden, dass Rita Maiburg für ihre Ausbildung als Pilotin 40000 Mark ausgegeben hat. Sie hat dieselbe Prüfung wie alle anderen Teilnehmer bestanden. Warum hat die Lufthansa trotz dessen so eine Entscheidung getroffen? Die Deutsche Lufthansa äußert sich hierzu folgendermaßen: Frauen sind zu anfällig, halten der Belastung nicht stand und sind im Hinblick auf die hohen Ausbildungskosten unrentabel, weil sie bald heiraten und dann ihren Beruf aufgeben (Deutsche Lufthansa).

Der Auffassung der Fluglinie ist zu entnehmen, dass keine Frau arbeiten sollte, weil sie dazu nicht fähig ist, das Privatleben mit dem Berufsleben zu verbinden. Dementsprechend ist nicht nur die Betroffene, sondern alle anderen Frauen sind benachteiligt.

\section{Entwicklung des Konflikts}

Nachdem der Grund der Zwietracht erläutert wurde, möchten wir nun auf die Reaktion der Frauenorganisation eingehen. Wie schon vorher erwähnt wurde, haben sich alle Frauen in der Situation diskriminiert gefühlt. Daraus resultierte der Protest, der auf den Straßen von Köln stattgefunden hat. Die Frauenorganisationen haben ihre Rechte manifestiert und gegen die Haltung der Deutschen Lufthansa protestiert. Die Demonstration beruhte darauf, dass die Frauen mit den Passanten gesprochen und Flugblätter verteilt haben. Hierzu einige Slogans: ,Männer fliegen - Frauen fliegen raus!'; ,Verfassungsfeinde in der Lufthansa!'; ,Wir fordern Gleichberechtigung!’.

Es ist natürlich nachvollziehbar, dass Frauen um ihre Rechte kämpfen wollen. Ob sie aber damit einen Kompromiss erzielten, ist im Moment unklar. 


\section{Gab es eine Konfliktlösung?}

In erster Linie ist zu fragen, ob die Fluglinie sich tatsächlich zu dem Protest der Frauen geäußert hat? Die Tatsache ist, dass die Lufthansa die Polizei über die Demonstration informiert hat. Wegen Verdacht gegenüber den Frauen, das Gebäude zu besetzen, rückte die Polizei zur Verteidigung des Lufthansabüros an. Letztendlich rückte sie wieder ab, da kein Eingriff seitens der Polizei nötig war.

Aus den gemachten Beobachtungen lässt sich folgendes Resümee ziehen: Den Ausgangspunkt für diese Auseinandersetzung war die Absage an Rita Maiburg, als Pilotin bei Lufthansa eingestellt zu werden. Die Begründung der Lufthansa war, die Frauen seien nicht imstande die Arbeitsbedingungen u. a. wegen der Mutterschaft, zu erfüllen. Meiner Meinung nach ist es ein Unsinn, da viele andere Frauen die Rolle der Mutter mit den Arbeitspflichten in Einklang bringen können. Daraus resultierte der Protest der Frauenorganisation, die gezwungen waren, um ihre Rechte auf den Straßen von Köln zu kämpfen. Über eine Einigung zwischen den Konfliktparteien haben wir dennoch keine genaue Information.

\section{Text 2}

Frauen kämpfen um die Gleichberechtigung Einleitung

Mit der vorliegenden Arbeit wird die Absicht verfolgt, die Demonstration in Köln zu beschreiben. Im ersten Kapitel werden die Gründe für die Absage der Fluggesellschaft beschrieben. Ein weiterer Arbeitsschritt beschäftigt sich mit der Reaktion der Frauen auf die Anstellungsabsage. Im weiteren Verlauf der Arbeit widme ich mich der Kölner Polizei und ihrem Einsatz im Zentrum von Köln. Darüber hinaus wird die Entwicklung der Situation und ihre Beendigung beschrieben. Zunächst soll erklärt werden wie, es zu Protesten der Frauenorganisationen kam.

\section{Absagegründe}

Dieser Teil der Arbeit wird den Absagegründen gewidmet. Die Fluggesellschaft Lufthansa hat Rita Maiburg als zukünftige Pilotin abgelehnt. Die Vorsitzenden der Gesellschaft sind der Meinung, die Frauen seien anfällig und würden der Belastung nicht standhalten. Sie heiraten und werden oft schwanger kurz nach dem Arbeitsvertragsabschluss. Das könnte dann mit der Berufsausübung und den Aufgaben der Fluggesellschaft in Konflikt stehen.

\section{Reaktion der Frauen auf die Anstellungsabsage}

In diesem Teil werde ich mich mit den Reaktionen der Frauen auf die Anstellungsabsage befassen. Die Frauenorganisationen waren mit der Absage höchst empört und haben fast sofort mit der Demonstration reagiert. Sie versammelten sich vor dem Sitz der Lufthansa, d.h. im Zentrum Kölns. Sie führten Diskussionen mit Passanten, verteilten Flugblätter 
und spazierten mit den Spruchbändern, auf denen es solche Sprüche gab wie z.B. "Männer fliegen, Frauen fliegen raus". Hieraufverwiesen die Frauen mit dem Spruch: "Verfassungsfeinde in der Lufthansa" darauf, dass die Lufthansa gegen die Verfassung verstö $\beta$ t. Damit wollten die Frauen die Haltung der Lufthansa ans Tagelicht bringen und den Menschen das bewusst machen.

\section{Polizei im Einsatz}

Das dritte Kapitel stellt den durch Polizei durchgeführten Einsatz dar. Die Polizei rückte zum Schutz des Lufthansabüros an, aber nachdem sie sich orientiert hatten, dass es kein aggressives Verhalten gegeben hatte, rückte die Polizei wieder ab. Es stellte sich heraus, dass die Demonstrantinnen keine Absicht hatten, das Gebäude zu besetzen.

\section{Eskalation des Konflikts}

Im letzten Teil der Arbeit werde ich mich mit der Eskalation des Konflikts und dessen Ende beschäftigen. Es kam zur Eskalation des Konflikts, denn die Gewerkschaften haben sich eingemischt und in großem Ausmaß demonstriert. Die Lufthansa hat nach einiger Zeit eine offizielle Erklärung abgegeben, dass sie einen großen Fehler begann, indem sie Rita Maiburg ablehnten. Sie gaben zu, dass die Person, die Frau Rita Maiburg nicht eingestellt hat, disziplinär entlassen wurde und dass Frau Rita Maiburg eine Stelle als Pilotin bekommen wird, wenn sie nur die weitere Absicht hat, dort eine Arbeitsstelle aufzunehmen.

\section{Zusammenfassung}

Es lässt sich resümieren, dass es einen Fall gab, in dem die Lufthansa einer sich um die Stelle der Pilotin bewerbenden Frau abgesagt hat. Der Grund dafür war die Tatsache, dass der Bewerber eine Frau war. Die Frauen sind schwach, sie halten die Belastung nicht aus, heiraten schnell und folglich bekommen Kinder, was dazu führt, dass sie bald die Arbeit aufgeben. Diese Haltung der Fluggesellschaft war für die Frauenorganisationen unannehmbar, deshalb haben sie mit Demonstrationen geantwortet. Letztendlich kann man sagen, dass die ganze Sache sich so sehr verbreitet hat, dass sich die Lufthansa schnell zurückziehen musste, denn es konnte ihrem Image große Schäden bringen. Die Bewerberin hat ihre Traumstelle bekommen.

Bei der holistischen Evaluation fällt zunächst auf, dass die von den Studierenden angefertigten Texte fast vier Mal länger als der Ausgangstext sind ${ }^{13}$. Das bestätigt, dass manche Studierenden mit dem Ausgangstext kreativ und vor allem elaborativ umgegangen sind ${ }^{14}$. Die im Haupttitel und in den Überschriften

${ }^{13}$ Der Originaltext enthält 130 Wörter (Lexeme, Artikel, Präpositionen), die zitierten Texte 487 und 519 Vokabeln.

${ }^{14}$ Der Begriff elaborativ bezeichnet in der Psychologie die vertiefte Informationsverarbeitung. Durch Elaboration wird vorhandenes Vorwissen über einen Gegenstandsbereich aktiviert und neues Wissen mit diese [verknüpft (Irwin 1991: 89-108)]. 
angedeuteten Thesen wurden im Text entsprechend entwickelt und begründet. Die einzelnen Kapitel wurden mit einer kurzen Zielanagabe eingeleitet. Die Schreibergebnisse sind in dieser Hinsicht positiv zu bewerten.

Die große Fehleranzahl in den hier präsentierten Texten signalisiert, dass Studierende unbedingt auch Hinweise für die eigene, erfolgreiche Korrektur ihrer Arbeiten brauchen ${ }^{15}$. Die auffallenden Unsicherheiten machen deutlich, welche Kompetenzbereiche bei den Studierenden noch defizitär sind und einer gezielten Übung und Wissensvermittlung bedürfen. Somit hat sich die Schreibsimulation auch als ein zuverlässiges Mittel zur Erhebung des Kompetenzstandes im Bereich wissenschaftlichen Schreibens erwiesen. Die zitierten Texte eignen sich gut für die Einübung der Textkorrektur in einem Schreibseminar.

\section{FAZIT}

Der prozessorientierte Ansatz machte kognitive Ressourcen frei für Kreativität, Elaborationen und zusätzliche Textproduktion. Die dabei entstehende Belastung des kognitiven Systems schränkt jedoch die Aufmerksamkeit auf die Korrektheit der sprachlichen Formulierungen ein.

Ein systematischer Einsatz des hier vorgestellten Ansatzes kann zum erfolgreichen Erwerb von wissenschaftlichen Textprozeduren erheblich beitragen. Es ist auch denkbar, dass andere Teilfertigkeiten des wissenschaftlichen Schreibens auf ähnliche Weise vermittelt und eingeübt werden können. Die Diskussion der bei Schreibsimulationen entstehenden Probleme sowie der eigenen Schreibprodukte im Seminar kann das Interesse und Sensibilität für die hier behandelte Problematik unter den Studierenden steigern.

\section{LITERATURVERZEICHNIS}

Ballstaedt, S.-P. / Mandl, H. / Schnotz, W. / Tergan, S.-O. (1981). Texte verstehen, Texte gestalten. München / Wien / Baltimore: Urban \& Schwarzenberg.

Dietz, G. (1998). Titel in wissenschaftlichen Texten. In: L. Hoffmann / H. Kalverkämper / H.E. Wiegand / Ch. Galinski (Hrsg.), Fachsprachen. Languages for Special Purposes. Ein internationales Handbuch zur Fachsprachenforschung und Terminologiewissenschaft (S. 617-624). Berlin / New York: de Gruyter.

${ }^{15} \mathrm{Zu}$ Überarbeitungsstrategien des eigenen Textes gibt es für Studierende an deutschen Hochschulen bereits eine große Anzahl von Ratgeberliteratur (a.u. Edelmann 1995; Kruse 2014). Siehe auch UHH Tipps zum Schreiben. Überarbeiten. https:/ / www.slm.uni-hamburg.de/studium/ projekte/fremdsprachen-lernen/start-b/2-b-scr/b04.html\#3875117 [Zugriff am: 27.03.2021]. 
Edelmann, H. (1995). Textüberarbeitung. Revisionen in fremdsprachlichen Lerner-Texten (DaF). Prozesse der Überarbeitung narrativer, deskriptiver und argumentativer Texte in Lerner-Paaren. Frankfurt (a. M.): Lang.

Fischer-Kania, S. (2008). Die Förderung der Schreibfertigkeit in den DaF-Lehrwerken Delfin, em neu-Hauptkurs und Auf neuen Wegen. Info DaF, 35 (5), 481-517.

Gnutzmann, K. (1988). Aufsatztitel in englischsprachigen Fachzeitschriften. Linguistische Strukturen und kommunikative Funktionen. In: K. Gnutzmann (Hrsg.), Fachbezogener Fremdsprachenunterricht (S. 23-38). Tübingen: Narr.

Grammis. Deutsche Rechtschreibung. https://grammis.ids-mannheim.de/rechtschreibung [Zugriff am: 02.09.2020].

Grieshammer, E. (2011). Der Schreibprozess beim wissenschaftlichen Schreiben in der Fremdsprache Deutsch und Möglichkeiten seiner Unterstützung. Frankfurt (O.). https:/ / opus4.kobv.de/opus4euv/frontdoor/deliver/index/docId/49/file/Schreiben_im_Zentrum_3_Ella_Grieshammer. pdf [Zugriff am: 08.10.2020].

Hellwig, P. (1984). Titulus oder Über den Zusammenhang von Titeln und Texten. Titel sind ein Schlüssel zur Textkonstitution. ZGL, 12, 1-20.

Iluk, J. (2010). Empirische Befunde zur Auswirkung systematischer Schreibhandlungen im fortgeschrittenen FSU auf den Spracherwerb. Studia Germanica Gedanensia, 23, 23-37.

Iluk, J. (2015). Textorganisierende Redemittel als Forschungs-, Verwendungs-, und Lernproblem. In: R. Opiłowski / W. Czachur (Hrsg.), Sprache - Wissen - Medien. Festschrift für Professor Gerd Antos (S. 101-116). Wrocław / Dresden: Atut.

Iluk, J. (2021). Titel und Überschriften von Diplomarbeiten im ausländischen Germanistikstudium: Hintergrundwissen und Entwicklungsmöglichkeiten einer Titelgebungskompetenz. Beiträge zur Fremdsprachenvermittlung, 64, 6-20.

Irwin, J.W. (1991). Teaching reading comprehension processes. Second edition. Englewood Cliffs, NJ: Prentice Hall.

Karcher, G. (1988). Das Lesen in der Erst- und Fremdsprache. Dimensionen und Aspekte einer Fremdsprachenlegetik. Heidelberg: J. Gross.

Kohrs, J. (2010). In der Kürze liegt die Würze? Strategien der Informationsdarbietung in deutschen und litauischen Fachtiteln. Kolbatyra, 62 (3), 58-74.

Kruse, O. (2014). Lesen und Schreiben. Der richtige Umgang mit Texten im Studium. Konstanz: UVK.

Lüger, H.H. (1987). Titel und Textverstehen. Zur Rezeptionssteuerung in italienischen Gebrauchstexten. Italienisch, 17, 88-98.

Pospiech, U. (2004). Schreibend schreiben lernen - über die Schreibhandlung zum Text als Schreibwerk. Zur Begründung und Umsetzung eines feedbackorientierten Lehrgans zur Einführung in das wissenschaftliche Schreiben. ELISe, Beiheft 1. Essen: Universität Essen. https://www.uni-due.de/ imperia/md/content/elise/beiheft_01_2004.pdf [Zugriff am: 10.10.2020].

Püschel, U. (1997). Überlegungen zu einer Anleitung zum Schreiben von Hausarbeiten. In: E.-M. Jakobs / D. Knorr (Hrsg.), Schreiben in den Wissenschaften (S. 193-200). Frankfurt (a. M.): Lang.

Rettig, H. (2014). Leitfaden zur Erstellung von wissenschaftlichen Arbeiten am Institut für Germanistik. Koblenz: Campus Koblenz. https:/ / www.uni-koblenz-landau.de/de/koblenz/fb2/inst-germanistik/mitarbeiter/arend/infos/leitfadenha [Zugriff am: 10.10.2020].

Ruhmann, G. (1997). Ein paar Gedanken darüber, wie man wissenschaftliches Schreiben lernen kann. In: E.-M. Jakobs / D. Knorr (Hrsg.), Schreiben in den Wissenschaften (S. 125-139). Frankfurt (a. M.): UVK.

Rummer, R. / Mohr, G. / Zimmer, H. (1998). Leistungsbegrenzungen im verbalen Arbeitsgedächtnis: Argumente gegen energetische Ressourcenkonzepte. Kognitionswissenschaft, 7, 134-140. 
Sandig, B. (1997). Formulieren und Textmuster. Am Beispiel von Wissenschaftstexten. In: E.-M. Jakobs (Hrsg.), Schreiben in der Wissenschaft (S. 25-44). Frankfurt (a. M.): UVK.

Schäpers, R. / Luscher, R. / Glück, M. (1978). Deutsch 2000: eine Einführung in die moderne Umgangssprache. München: Huber.

Steinhoff, T. (2003). Wie entwickelt sich die wissenschaftliche Textkompetenz? Der Deutschunterricht, 3, 38-47.

Thonhauser-Jursnick, I. (2000). Wozu Schreiben im Fremdsprachenunterricht? Eine Analyse neuerer DaF-Lehrwerke. DaF, 4, 195-198.

Tipps zu Studientechniken und Lernmethoden (2011). Universität Bielefeld - Zentrale Studienberatung. https://www.uni-bielefeld.de/studium/studierende/information-studienberatung/studientechniken/literatur-links-angebote/Studientechniken.pdf [Zugriff am: 10.10.2020].

Venohr, E. (2009). Wissenschaftstraditionen, Stil und Bewerten bei der Vermittlung des Deutschen als Wissenschaftssprache. Didaktische Überlegungen im deutsch-französischen universitären Kontext. In: M. Dalmas / M. Foschi / E. Neuland (Hrsg.), Wissenschaftliche Textsorten im Germanistikstudium deutsch-italienisch-französisch kontrastiv. Trilaterales Forschungsprojekt in der Villa Vigoni 2007-2008, Band 2 (S. 294-306). Frankfurt (a. M.): Lang.

Venohr, E. (2013). Didaktische Aspekte fremdsprachlicher Textproduktion in der Wissenschaftssprache Deutsch. In: F. Grucza et al. (Hrsg.), Band 16: Akten des XII. Internationalen Germanistenkongresses, Warschau 2010. Germanistische Textlinguistik. Digitalität und Textkulturen. Vormoderne Textualität. Diskurslinguistik im Spannungsfeld von Diskription und Kritik (S. 175-181). Frankfurt (a. M.): Lang.

Wysocka, M. (1989). Rozwój umiejętności mówienia i pisania w języku angielskim. Katowice: Prace Naukowe Uniwersytetu Śląskiego.

Received: 20.10.2020; revised: 09.04.2021

JAN ILUK

Uniwersytet Śląski w Katowicach

jan.iluk@us.edu.pl

ORCID: 0000-0003-2539-1936 